\title{
Aspects méthodologiques \\ de la prévision de la digestibilité de l'herbe pâturée par le mouton, les bovins et le cheval à partir de bols de l'œsophage et de diverses caractéristiques fécales
}

\author{
M. CHENOST \\ avec la collaboration technique de J.M. BoISSAU, \\ $M^{\text {miss JAILLER }}$ et JAMOT et L. L'Hotelier \\ INRA, Laboratoire des Aliments \\ C.R.Z.V. de Theix, F 63122 Ceyrat
}

\section{Résumé}

Cette étude a pour but d'analyser certains aspects méthodologiques de la prévision de la digestibilité de l'herbe au pâturage chez trois espèces animales (mouton, bovins, cheval), à partir des caractéristiques des contenus œsophagiens (bols) ou des fèces émises. Elle a été réalisée à partir de trois essais destinés par ailleurs à comparer la digestibilité, mesurée à l'auge, de l'herbe verte chez le mouton, les bovins et Ie cheval.

Le taux de récupération (fraction de l'herbe ingérée passant à travers la fistule de l'œsophage) n'est pas amélioré lorsqu'on obstrue l'oesophage en aval de la fistule. Il est plus faible chez le ruminant que chez le poney. On a pu, cependant, recueillir 3 à $500 \mathrm{~g}$ (matière brute) de bols en 20 minutes, quantités comparables à celles observées par les autres auteurs. La composition, la digestibilité in vitro (DIV) et la digestibilité cellulase (après attaque à la pepsine-cellulase) des bols ne sont pas affectées par la présence de salive. La DIV et la digestibilité cellulase des bols œesophagiens sont plus élevées que celles de l'échantillon représentatif du fourrage proposé, elles-mêmes plus élevées que la digestibilité mesurée in vivo. Il est donc impossible de déduire la digestibilité in vivo à partir de la DIV ou la digestibilité cellulase des bols sans avoir recours à une équation de régression dont la précision, peu élevée (Syx $<3$ points), est meilleure (sauf pour la digestibilité cellulase chez le mouton), lorsque les bols sont séchés à l'étuve que lorsqu'ils sont lyophilisés.

La digestibilité in vivo peut en revanche être prévue avec une meilleure précision à partir des caractéristiques fécales. Ces dernières sont pariétales : cellulose brute (CBF), non pariétales : teneur en matières azotées totales (MATF), matières solubles dans l'eau (MSE) ou physiques : densité (DENS), granulométrie (GRAN); ces caractéristiques étant prises seules ou deux à deux. Cette précision, comprise en moyenne entre 2 et 3 points, est la meilleure avec MATF et GRAN chez le mouton (Syx $=2,16)$, avec MSE et DENS chez le cheval (Syx $=2,16$ ) et avec MATF et MSE (Syx $=1,95)$ chez les bovins.

Les équations, basées sur les caractéristiques soit des bols nesophagiens soit des fèces ont été appliquées aux mesures effectuées sur des moutons et des chevaux mis simultanément au pâturage au cours du $1^{\mathrm{er}}$ et $2^{\mathrm{e}}$ cycle de repousse d'une prairie naturelle. Elles conduisent à des valeurs de la digestibilité de l'herbe pâturée très voisines et regroupées pour le mouton, 
1rès dispersées pour le cheval. Elles permettent de mettre en évidence le tri effectué par le mouton au $2^{\prime \prime}$ cycle.

Les avantages respectifs des deux groupes de méthodes sont discutés; ils doivent être considérés avec prudence, compte tenu du nombre de mesures encore limité.

Mots clés : méthodologie, digestibilité, pâturage, bols, fèces.

\section{Introduction}

La prévision de la digestibilité de l'herbe pâturée par les ruminants a fait l'objet de très nombreux travaux depuis les années 1950. Ces derniers ont été analysés de manière critique dans plusieurs revues (STREeTER, 1969 ; Langlands, 1975 ; Meijs, 1981 ; LE Du \& Penning, 1982). Il existe diverses techniques pour prédire la digestibilité ; chacune présente ses avantages et ses inconvénients propres qui dépendent de l'espèce et du type de l'animal ainsi que de la nature et du mode d'exploitation de la prairie.

Parmi les méthodes «directes», l'utilisation des liaisons existant entre les caractéristiques chimiques et physiques des fèces et la digestibilité de l'herbe est intéressante. Elle permet de prévoir la digestibilité avec une bonne précision surtout lorsqu'on prend en compte les caractéristiques physiques en plus de leurs caractéristiques chimiques (CHENOST, 1985). Cette méthode suppose toutefois l'établissement préalable d'équations de régression digestibilité/caractéristiques fécales propres à chaque prairie (voire à chaque cycle de repousse). Elle est donc lourde.

La collecte d'échantillon d'herbe pâturée à travers une fistule de l'œsophage pratiquée sur des animaux introduits dans la parcelle à étudier, est séduisante. On peut estimer directement la digestibilité de l'herbe grâce à une mesure de digestibilité in vitro, in sacco ou encore à la pepsine/cellulase du bol œsophagien recueilli. Cette technique suppose toutefois des interventions chirurgicales et l'entretien des animaux porteurs de canules.

L'objet de notre étude est d'analyser certains aspects méthodologiques liés à ces deux techniques afin d'en étudier les intérêts et inconvénients respectifs chez trois espèces animales.

\section{Matériel et méthodes}

\section{A. Dispositif expérimental}

Nous avons réalisé trois essais $(1980,1981$ et 1982) destinés par ailleurs à comparer la digestibilité et les quantités d'herbe verte ingérées à l'auge chez le mouton, le bovin et le cheval (CHENost \& MARTin-Rosset, 1985). Nous rappelons dans le tableau 1 les principaux éléments du dispositif expérimental utilisé.

L'herbe a été exploitée en affouragement en vert à chaque essai. Elle a été distribuée sous forme longue dans le premier essai (à des vaches laitières, des 


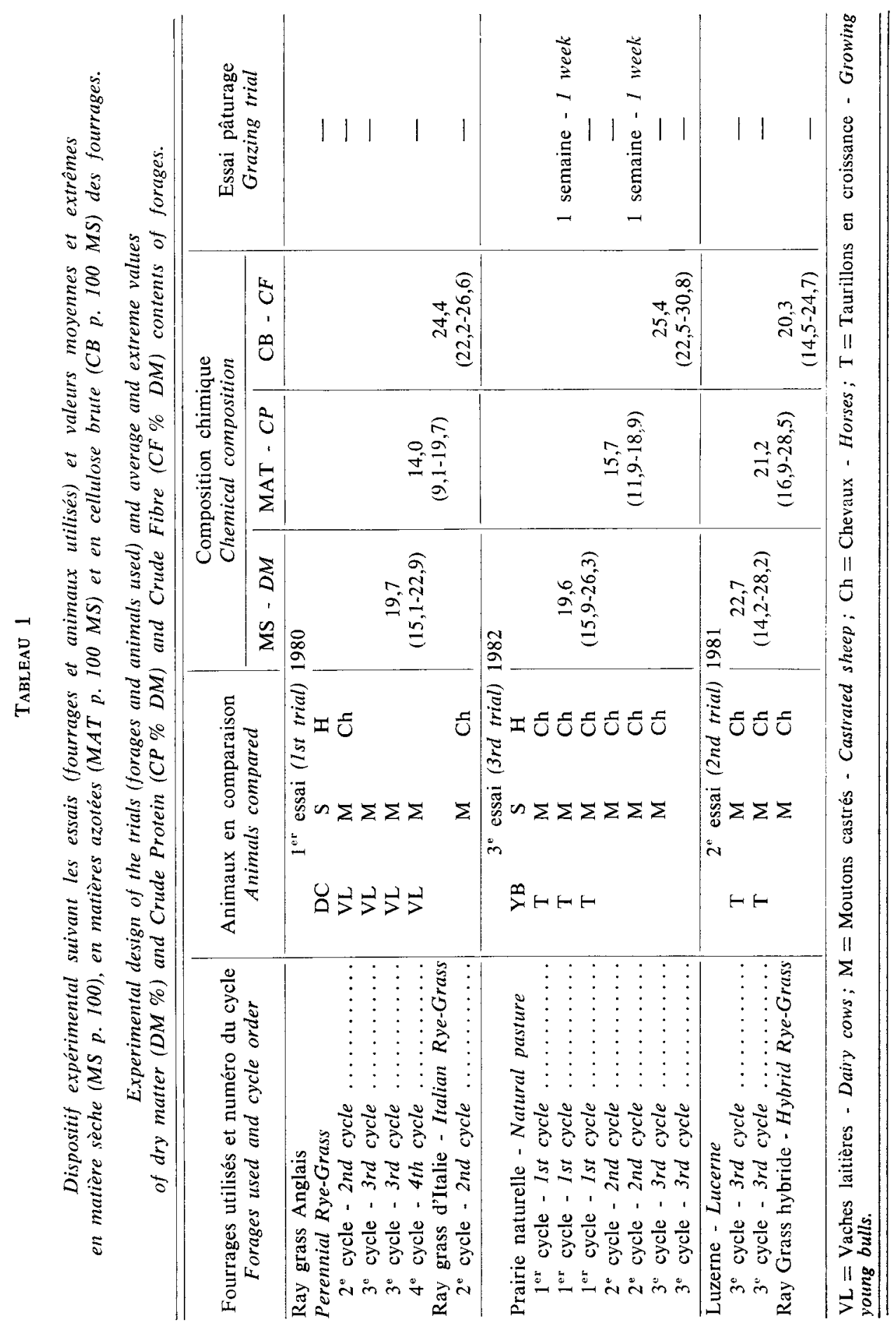


moutons et des chevaux - trotteurs réformés), et sous forme hachée (ensileuse à coupe fine) dans les deux autres essais (à des moutons, des taurillons et des trotteurs). Les modalités de fauche et de distribution (ad libitum, aucun concentré) de l'herbe, ainsi que les techniques de mesure de la digestibilité et des quantités ingérées ont été décrites antérieurement (Chenost \& MARTin-Rosset, 1985).

On a mesuré les quantités d'herbe ingérées et la digestibilité à l'auge sur les moutons, les taurillons, les vaches laitières et les trotteurs alimentés à volonté (deux repas par jour, à $9 \mathrm{~h}$ et à $16 \mathrm{~h}$ ) pendant les trois essais (tabl. 1).

Au cours du $2^{\mathrm{e}}$ essai une partie de la prairie a été pâturée simultanément lors du $1^{\text {er }}$ et du $2^{\circ}$ cycle par deux pouliches de 2 ans (pesant chacune $500 \mathrm{~kg}$ ) et 6 moutons adultes (pesant chacun $60 \mathrm{~kg}$ ). Les surfaces accordées ont été calculées de manière à respecter à chaque cycle la même pression du pâturage. Cette dernière permettait une alimentation à volonté avec un pourcentage de refus (10 à 15 p. 100) voisin de celui adopté pour l'alimentation à l'auge. Il ne nous a pas été possible d'utiliser des bovins au pâturage.

\section{B. Collecte et conditionnement des bols de l'osophage et des fèces à l'auge et au pâturage}

\section{Bols de l'œesophage}

Nous avons utilisé 4 moutons mâles castrés, 2 génisses de 2 ans et 1 poney fistulés de l'œsophage et équipés d'une canule pleine amovible. Nous n'avons pas fistulé de chevaux mais un poney car, même si la technique opératoire était bien maîtrisée, nous connaissions mal la façon dont un cheval pourrait supporter ultérieurement sa fistule. Les moutons (maintenus sur caillebotis en case au sol), les génisses (en stabulation entravée) et le poney (en box) recevaient la même herbe que les animaux en digestibilité suivant la séquence indiquée dans le tableau 1.

On a recueilli les bols alimentaires à trois reprises (lundi, mercredi, vendredi) pendant les semaines de mesure de la digestibilité de façon à constituer un échantillon représentatif du fourrage offert par période de mesure. Après retrait de la canule œsophagienne, des sacs de plastique de taille et de forme adaptées à l'animal (ouventure du sac sur les $2 / 3$ de sa longueur à partir du haut) étaient fixés à l'aide d'épingles par enroulement des deux parties libres ainsi obtenues autour du cou de l'animal mis préalablement à jeûn. Cette opération était effectuée au début des grands repas $\mathrm{du}$ matin $(9 \mathrm{~h})$ ou de l'après-midi $(16 \mathrm{~h})$. Les sacs de collecte étaient maintenus en place pendant 20 à 30 minutes pour obtenir une quantité suffisante de matière fraîche (300 g chez le mouton, 500 à $1000 \mathrm{~g}$ chez le poney et les génisses) et pour éviter un rétrécissement trop important de la lumière de la fistule et, par là, des difficultés dans la remise en place de la canule.

$\mathrm{Au}$ cours du $1^{\text {er }}$ essai nous avons étudié si le fait d'obturer la lumière de l'œsophage en aval de la fistule avait une influence, d'une part sur le taux de récupération des bols (quantité d'herbe (MS) passant à travers la fistule, rapportée à la quantité de matière sèche ingérée), d'autre part sur la qualité des bols (influence éventuelle de la salive sur leur composition et leur digestibilité).

Pendant les deux périodes de pâturage du $2^{\mathrm{e}}$ essai le lot de 6 moutons était constitué de 4 moutons normaux et de 2 fistulés de l'œsophage. Nous avons 
utilisé le poney fistulé en le conduisant à jeûn sưr la prairie où il pâturait pendant une demi-heure avant de regagner son box. On a procédé à la collecte des bols de la même façon qu'à l'auge.

Les bols frais ainsi recueillis ont été soumis à différents modes de conditionnement :

- pressage ou non dans une gaze pour éliminer la salive ;

- séchage à l'étuve à $80^{\circ}$ pendant 48 h., ou congélation suivie d'une lyophilisation.

\section{Fèces}

Les fèces des animaux à l'auge ont été collectées suivant la méthode classique d'échantillonnage utilisée pour les mesures de digestibilité. Les fèces des animaux au pâturage ont été collectées :

- à l'aide de bidons et de harnais chez les moutons (Trssier et al., 1975) ;

- par prélèvements au sol de la totalité des fèces émises par les pouliches.

Dans chacun des deux cas (auge et pâturage) une partie des fèces fraîches a été mise au congélateur sous forme non broyée et l'autre mise à sécher à l'étuve à $80^{\circ} \mathrm{C}$.

\section{Analyses}

On a effectué les analyses et mesures suivantes en laboratoirc :

- sur les échantillons représentatifs de l'herbe offerte pendant la semaine de mesure de digestibilité, les bols de l'œsophage correspondants et les bols récoltés au pâturage : teneur en matière sèche (MS), en cendres, en matières azotées totales (MAT), en cellulose brute (CB), résidus d'attaque au détergent neutre et acide (NDF et ADF) de VAN Soest (1967), digestibilité in vitro de TILley \& TerRy (1963) (DIV), digestibilité après attaque à la cellulase selon Aufrere (1982) (digestibilité cellulase);

- sur les échantillons représentatifs des fèces (auge et pâturage) : teneur en MS, en MATF, en matières solubles dans l'eau (MSEF) pour les critères non pariétaux ; teneur en $\mathrm{CB}$, en NDF et ADF pour les critères pariétaux ; enfin deux caractéristiques physiques, la densité (DENS) d'après JARRIGE (1965) et la granulométrie (GRAN) selon GRENET (1966). La granulométrie a été mesurée sur les fèces décongelées non broyées.

\section{II. Résultats et discussion}

La teneur en matières azotées totales (MAT) de l'herbe a varié de 11,9 à 28,5 (p. 100 MS) (tabl. 1). La digestibilité de la matière organique de l'herbe, mesurée à l'auge, a varié de 57,0 à 74,9 ; de 50,9 à 71,4 et de 67,8 à 83,1 p. 100 respectivement, chez le mouton, le cheval et les bovins (Chenost \& MARTin-Rosset, 1985). 


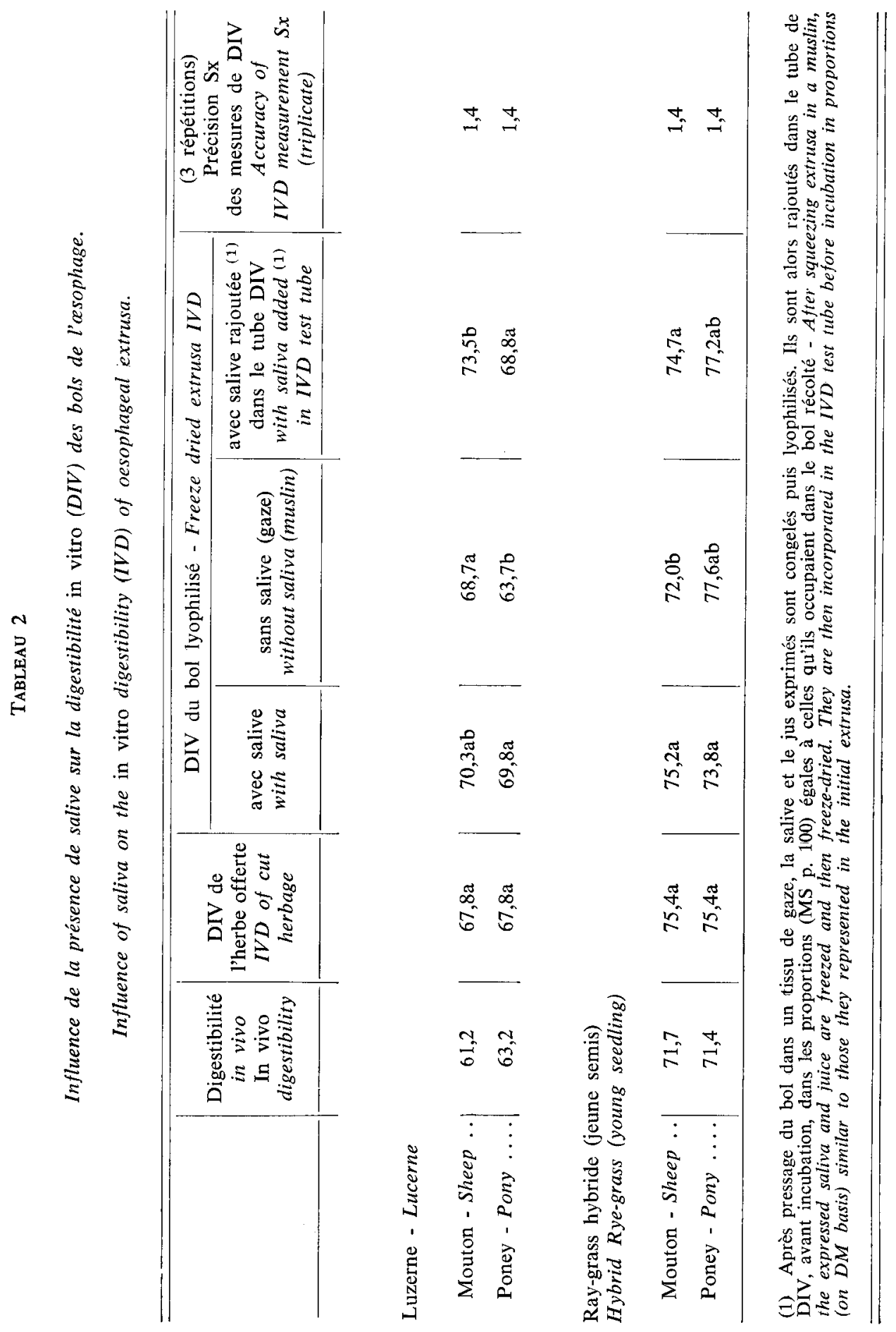




\section{A. Aspects méthodologiques relatifs aux bols oesophagiens}

\section{Collecte des bols}

L'obstruction de l'œsophage a modifié le comportement des animaux. Ils mangeaient de manière intermittente et salivaient abondamment. La quantité d'herbe récoltée par rapport à celle finalement ingérée restait faible et les bols recueillis étaient surtout constitués de salive. Aussi avons-nous décidé de ne plus obturer l'œsophage pour la suite des essais.

Une fois les animaux accoutumés à leurs fistules et habitués à être manipulés nous pouvions récolter des bols de 300 à $500 \mathrm{~g}$ en 20 à 30 minutes, quantités comparables à celles généralement observées par la plupart des auteurs.

La teneur en matière sèche des bols œsophagiens a été en moyenne égale à la moitié de celle du fourrage offert (présence d'un minimum de salive). Elle est plus élevée chez le poney (12,8 p. 100) que chez les moutons $(8,7$ p. 100) et les bovins $(8,1$ p. 100$)$, sa variabilité ne dépend pas de l'espèce animale.

\section{Conditionnement des bols (préparation, séchage)}

Les bols œesophagiens ont été récoltés directement sans éliminer la salive pendant les trois essais. Au cours du $3^{\mathrm{e}}$ essai nous avons toutefois vérifié sur 2 échantillons de fourrage si le fait de presser le bol dans une gaze avait un effet sur sa digestibilité in vitro. Le tableau 2 montre que la digestibilité in vitro du bol pressé (sans salive) diminue dans 3 cas sur 4 mais de manière variable suivant le fourrage et l'espèce animale (il diminue dans le cas d'un échantillon " poney »).

Le mode de séchage n'a pas modifié la composition chimique des bols de l'œsophage. La teneur en cendres a été de $12,5-12,5$ et 13,8 et la teneur en MAT de $17,3-17,6$ et 17,5 respectivement pour les échantillons d'herbe offerte, les bols « moutons » séchés à l'étuve et les bols «moutons " lyophilisés. Il en a été de même pour les bols « poney» et «bovins».

Ces résultats permettent, en même temps, de constater que la présence de salive n'a pas augmenté la teneur en cendres de manière significative, contrairement aux observations classiques (LE DU \& Penning, 1982).

En revanche (fig. 1) la digestibilité in vitro des bols lyophilisés récoltés chez les moutons a toujours été supérieure (de 2,6 points en moyenne) à la digestibilité in vitro des bols séchés à l'étuve. Cette dernière est, à 5 exceptions près sur 15 , supérieure à la digestibilité in vitro des échantillons représentatifs de l'herbe offerte aux animaux pendant les périodes de mesure de digestibilité. Les écarts entre la digestibilité in vitro des bols séchés et celle des bols lyophilisés présentent une grande variabilité (fig. 1). On observe ces mêmes différences pour la digestibilité cellulase (fig. 2). Les variabilités dues au mode de séchage sont encore plus prononcées, dans le cas des bols œesophagiens recueillis chez le poney, particulièrement pour la digestibilité in vitro. 


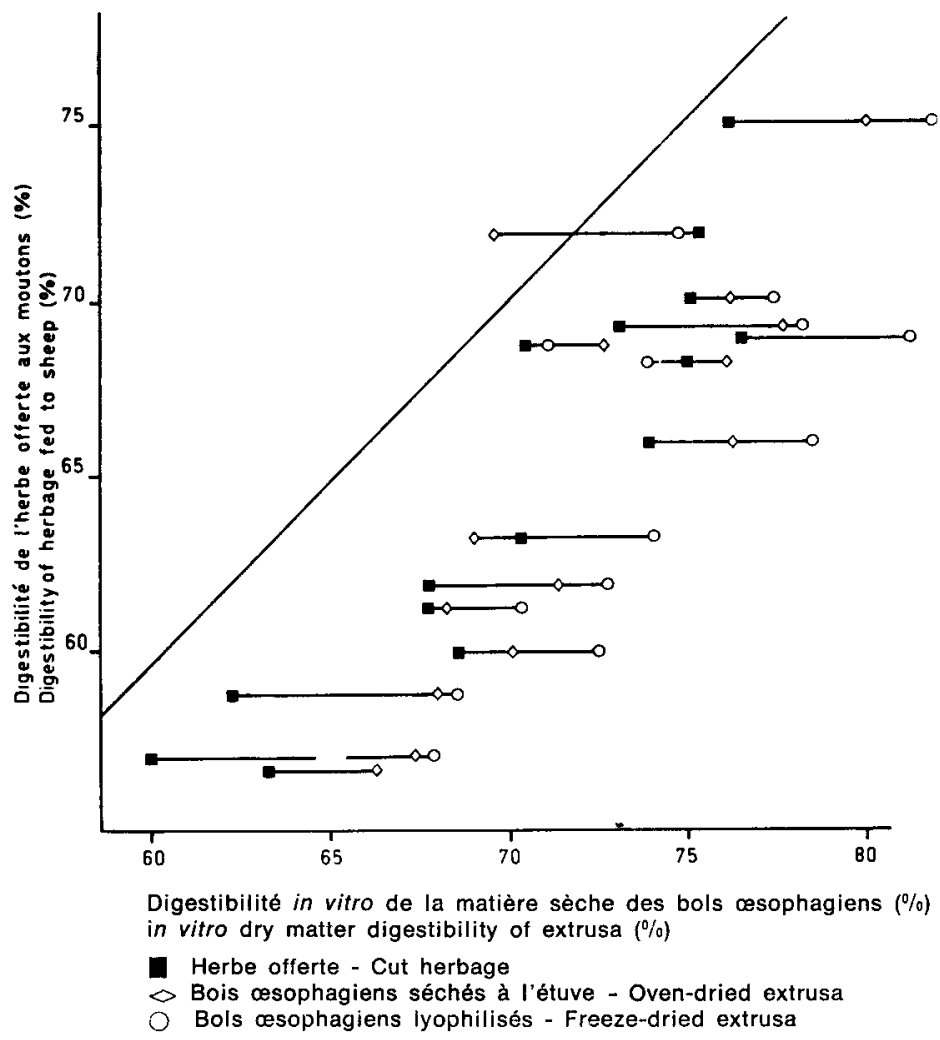

Fig. 1

Influence du mode de séchage des bols asophagiens de mouton sur leur digestibilité in vitro suivant la digestibilité de l'herbe offerte.

Comparaison avec la digestibilité in vitro de l'échantillon représentatif de l'herbe offerte.

Influence of the drying method of sheep extrusa on their in vitro digestibility (DM.IVD) according to herbage digestibility (DMD).

Comparison with the in vitro dry matter digestibility of the representative sainple of herbage.

B. Liaisons entre la digestibilité de la matière sèche de l'herbe distribuée à l'auge et les digestibilités in vitro et cellulase des bols cesophagiens (mouton-poney)

Les figures 1 et 2 montrent que la digestibilité in vitro des échantillons représentatifs de l'herbe proposée (DIV.P) est en liaison étroite avec la digestibilité in vivo (dms) mesurée sur moutons,

$$
\mathrm{dms}=179,2-4,33 \text { DIV.P }+0,038 \text { DIV.P } \mathrm{P}^{2} \pm 2,33 \mathrm{R}=0,923 \mathrm{n}=15,
$$

et avec la digestibilité in vivo mesurée sur cheval,

$$
\mathrm{dms}=43,3-0,27 \text { DIV.P }+0,0075 \text { DIV.P } \mathrm{P}^{2} \pm 3,73 \mathrm{R}=0,830 \mathrm{n}=9 .
$$


Il en est de même avec la digestibilité cellulase (D. Cellulase P.), $\mathrm{dms}$ (mouton) $=0,23+1,05 \mathrm{D}$. Cellulase $\mathrm{P}-0,002 \mathrm{D}$. Cellulase $\mathrm{P}^{2} \pm 2,31$

$$
\mathrm{R}=0,900 \quad \mathrm{n}=13
$$

et

$$
\begin{gathered}
\text { dms }(\text { cheval })=114,40+4,20 \text { D. Cellulase } P-0,025 \text { D. Cellulase } P^{2} \pm 1,24 \\
R=0,970 \quad n=7
\end{gathered}
$$

Ces liaisons sont les plus étroites avec la DIV chez le mouton et avec la digestibilité cellulase chez le cheval.

Comme les digestibilités in vitro et "cellulase " des bols œsophagiens s'écartent des digestibilités in vitro et in vivo de l'herbe offerte (fig. 2), et cela de manière différente suivant le mode de séchage des bols œsophagiens, il apparaît nécessaire de calculer des équations de régression liant ces digestibilités à la digestibilité de l'herbe offerte. Nous avons regroupé ces équations dans le tableau 3.

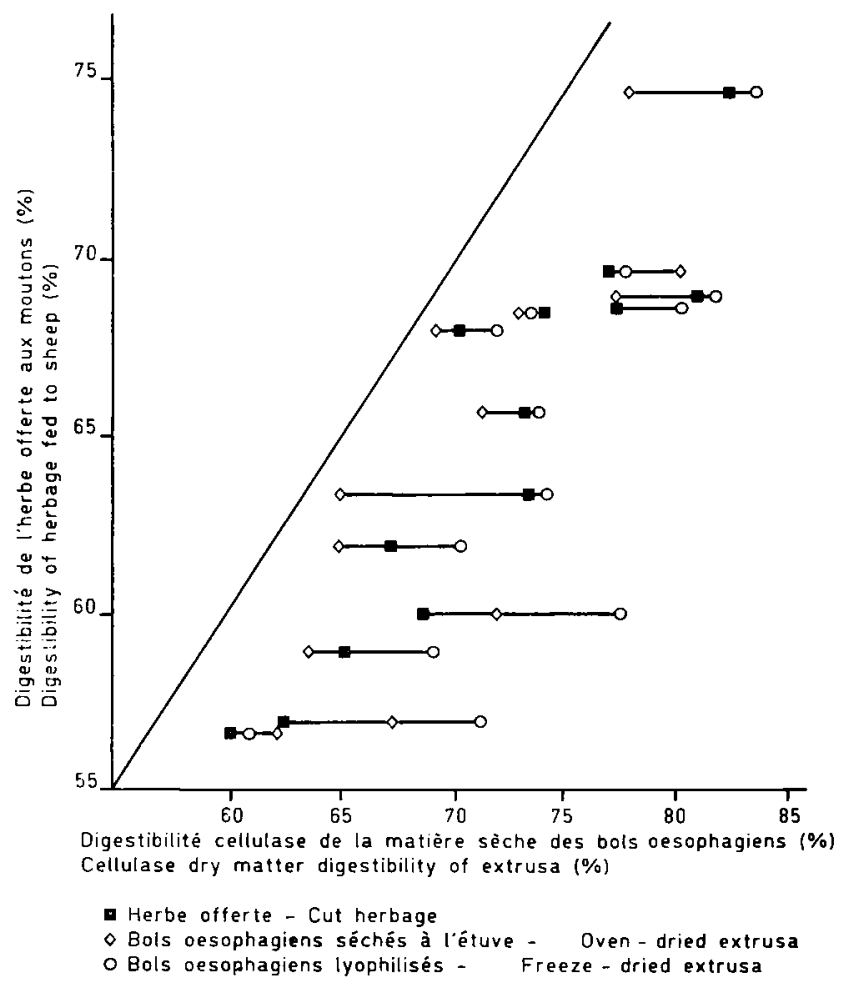

FIG. 2

Influence du mode de séchage des bols asophagiens de mouton sur leur digestibilité cellulase suivant la digestibilité de l'herbe offerte.

Comparaison avec la digestibilité cellulase de l'échantillon représentatif de l'herbe offerte.

Influence of the drying method of sheep extrusa on their cellulase digestibility according to herbage digestibility (DMD).

Comparison with the cellulase digestibility of the representative sample of herbage. 


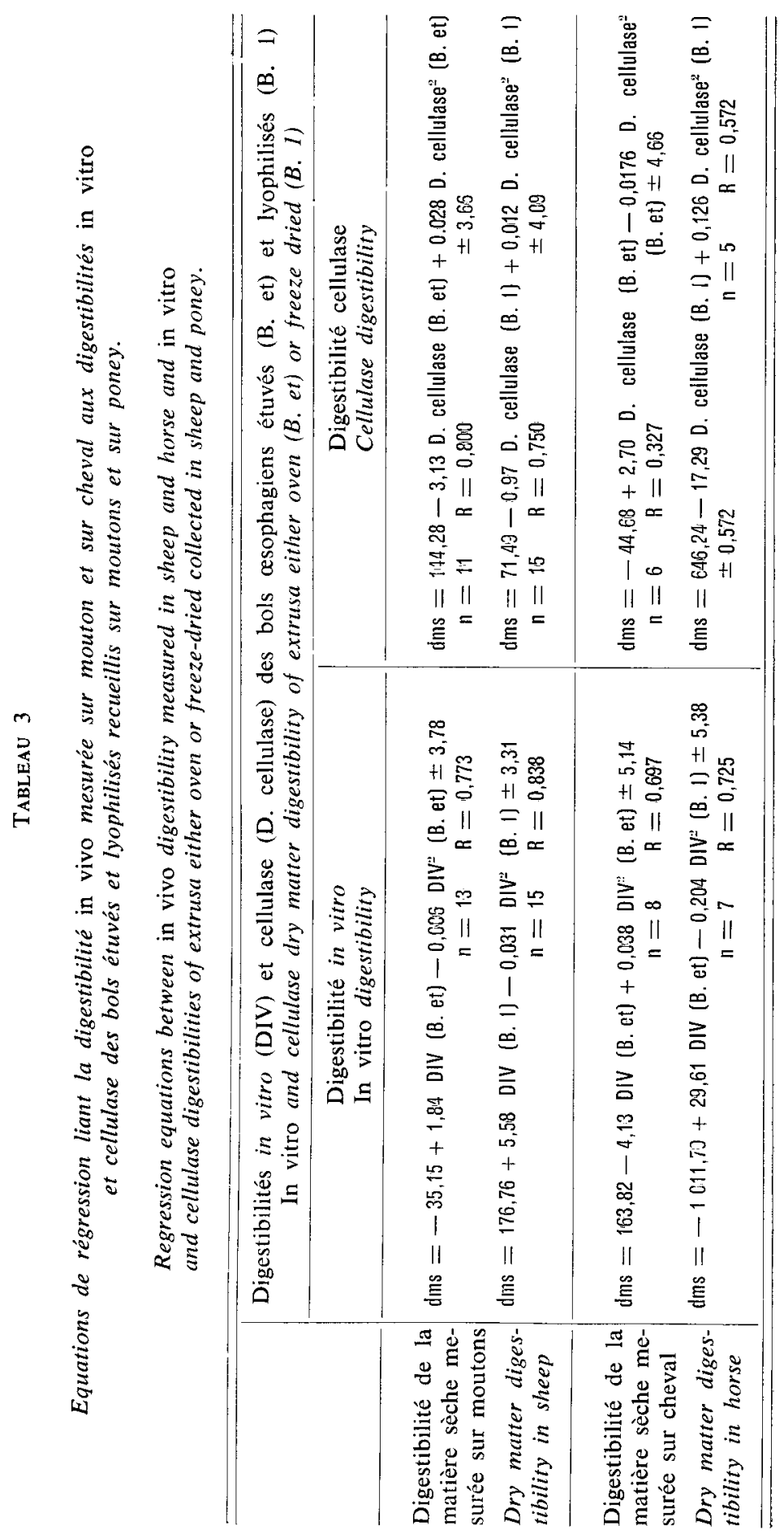


Ces dernières sont toutes du $2^{r}$ degré. Les liaisons entre la DIV des bols et la digestibilité (dms) sont plus étroites chez le mouton que chez le cheval. La prévision de la digestibilité à partir de la DIV est plus précise avec la lyophilisation qu'avec le séchage à l'étuve chez le mouton contrairement à ce qu'on observe chez le cheval. Les liaisons entre la digestibilité cellulase des bols et dms sont également plus étroites chez le mouton que chez le cheval. Le séchage à l'étuve conduit à une meilleure précision que la lyophilisation chez les deux espèces animales.

L'ensemble de ces relations montre qu'on ne peut pas, comme le font de nombreux auteurs, utiliser directement les équations dms/digestibilité in vitro ou cellulase de l'herbe proposée pour prévoir la digestibilité de l'herbe à partir des valeurs «in vitro» ou "cellulase" des bols cesophagiens.

\section{Liaisons entre la digestibilité de la matière organique de l'herbe distribuée à l'auge et les caractéristiques fécales}

Lors d'une étude précédente (CHENOST, 1985), nous avions vérifié sur 46 échantillons de fèces de moutons pris parmi 1230 fèces résultant de la mesure de la digestibilité des diverses espèces prairiales françaises, que le fait d'ajouter d'autres caractéristiques fécales à la teneur en matières azotées des fèces (MATF) permettait d'améliorer la relation liant MATF à la digestibilité.

Nous avons calculé, pour chaque espèce animale, les équations liant la digestibilité in vivo de la matière organique (dMO) aux différentes caractéristiques fécales prises une à une ou associées deux à deux. La précision des liaisons est reportée dans le tableau 4. On a éliminé NDF et ADF des calculs après avoir vérifié graphiquement que ces caractéristiques ne présentaient pas de liaisons significatives avec la dMO.

\section{Caractéristiques fécales considérées seules}

La précision de la prévision de la dMO mouton est bonne puisque, à l'exception de CBF ( $\mathrm{Sx}=4,50 ; \mathrm{r}=0,391$ ), l'erreur obtenue avec chacune des quatre variables est comprise entre 2,26 et 3,05 points de digestibilité pour 10 échantillons, MATF étant le meilleur critère de prévision. La précision de la prévision de dMO cheval varie de 2,96 à 4,48 points. Elle est moins bonne que dans le cas du mouton. La teneur en MATF est également le critère de prévision le plus précis. Les résultats bovins sont différents de ceux obtenus sur moutons et sur cheval. MATF, DENS et GRAN ne présentent pas de liaisons significatives avec la dMO. En revanche, c'est MSEF qui permet la meilleure prévision $(1,90)$ et qui se classe $1^{\text {r" }}$ variable explicative, CBF ne permet qu'une précision de 4,58 points.

\section{Caractéristiques fécales prises deux à deux}

Les variables prises 2 à 2 permettent une meilleure prévision que les variables prises seules. Cependant chez le mouton la combinaison permettant la meilleure prévision (MATF, GRAN), n'apporte que peu d'amélioration (Syx $=2,16$ ) par rapport à MAT seule $(S y x=2,26)$. La précision est comprise entre 2 et 2,5 points de digestibilité, sauf pour MATF, CBF et MSEF, DENSF. L'ensemble des deux caractéristiques physiques (DENS, GRAN) qui n'impliquent que des mesures simples conduit à un bon degré de prévision (2,5 points de digestibilité). 


\section{TABLEAU 4}

Liaisons, suivant l'espèce animale,

entre la digestibilité de la matière organique (dmo) de l'herbe et diverses caractéristiques fécales (1).

Relationship, according to animal species, between organic matter digestibility of herbage and various faecal characteristics (1).

\begin{tabular}{l|cc|cc|cc}
\hline \hline $\begin{array}{c}\text { Caractéristiques } \\
\text { fécales } \\
\begin{array}{c}\text { Faecal } \\
\text { characteristics }\end{array}\end{array}$ & \multicolumn{2}{|c|}{ Mouton - Sheep } & \multicolumn{2}{c|}{ Cheval - Horse } & \multicolumn{2}{c}{ Bovins - Cattle } \\
\cline { 2 - 3 } & $\mathrm{R}$ & Syx & $\mathbf{R}$ & Syx & $\mathbf{R}$ & Syx \\
\hline
\end{tabular}

a) Une variable - One variable

\begin{tabular}{ll|l|ll|lc} 
MATF $\ldots \ldots \ldots$ & 0,886 & 2,26 & 0,854 & 2,96 & 0,269 & NS \\
DENSF $\ldots \ldots \ldots$ & 0,850 & 2,57 & 0,758 & 3,59 & - & NS \\
MSEF $\ldots \ldots \ldots$ & 0,811 & 2,86 & 0,615 & 4,48 & 0,926 & 1,90 \\
GRAN $\ldots \ldots \ldots$ & 0,781 & 3,05 & 0,820 & 3,26 & - & NS \\
CBF ......... & 0,391 & 4,50 & 0,597 & 4,42 & 0,408 & 4,58
\end{tabular}

b) Deux variables - Two variables

\begin{tabular}{l|ll|ll|lc} 
MATF, GRAN . . & 0,910 & 2,16 & 0,906 & 2,67 & - & - \\
MATF, MSEF . & 0,900 & 2,27 & 0,887 & 2,84 & 0,933 & 1,95 \\
MSEF, cendres . & 0,899 & 2,28 & 0,879 & 2,93 & 0,316 & NS \\
MATF, DENSF . & 0,890 & 2,38 & 0,929 & 2,17 & 0,337 & NS \\
MATF, cendres . & 0,889 & 2,39 & 0,917 & 2,35 & 0,316 & NS \\
GRAN, DENSF . & 0,877 & 2,50 & 0,902 & 2,65 & - & - \\
MSEF, GRAN . . & 0,872 & 2,55 & 0,862 & 3,11 & - & - \\
MATF, CBF . . & 0,589 & 4,60 & 0,801 & 3,33 & 0,416 & NS \\
MSEF, DENSF . & 0,555 & 4,95 & 0,936 & 2,16 & - & - \\
\hline
\end{tabular}

(1) MATF : Teneur en matières azotées totales (p. 100).

Crude protein content ( $p .100$ DM).

MSEF : Teneur en matières solubles dans l'eau (p. 100 MS).

Water soluble matter (p. 100 DM).

DENSF : Densité (JARRIGE, 1965).

Specific gravity.

GRAN : Granulométrie (GReNET, 1966). Particle size.

CBF : Teneur en cellulose brute (p. 100 MS).

Crude fiber content (p. 100 DM).

Cendres : Teneur en cendres (p. 100 MS).

Ash content (p. 100 DM). 
Chez le cheval, les résultats sont différents de ceux obtenus chez le mouton mais l'erreur de prévision est du même ordre. La combinaison permettant la meilleure prévision $(2,16$ points de $\mathrm{dMO})$ est l'ensemble des deux variables « physiques " (DENS, GRAN). Ceci peut s'expliquer par le caractère plus fibreux des fèces du cheval que du ruminant. En effet, plus un fourrage vieillit, plus ses parois se lignifient et deviennent difficiles à réduire en fines particules, particulièrement chez le cheval dont l'efficacité de mastication et l'activité cellulolytique sont plus faibles que chez le ruminant.

Les liaisons obtenues sur bovins ne sont pas significatives à l'exception de MSEF et de la combinaison MATF, MSEF qui permettent une bonne prévision. Cela est vraisemblablement dû au fait que nous avons travaillé à la fois sur vaches laitières et sur taurillons en croissance.

D. Application pratique : valeur estimée de la composition chimique (MAT p. $100 \mathrm{MS}$ ) et de la digestibilité de l'herbe pâturée au $1^{\text {er }}$ et au $2^{e}$ cycle par le mouton et le cheval

- Comparaisons, suivant le numéro du cycle et l'espèce animale, des caractéristiques (MAT, dMS) des bols cesophagiens récoltés d'une part à l'auge et d'autre part au pâturage : essai d'appréciation du tri au pâturage :

Comme nous l'avons constaté plus haut la teneur en MAT des bols collectés sur les animaux à l'auge (mouton et poney, séchage à l'étuve et lyophilisation) n'est pas significativement différente de celle de l'herbe offerte. Il est donc vraisemblable que, de la même façon, la teneur en MAT des bols collectés sur les animaux au pâturage ne soit pas différente de celle de l'herbe effectivement pâturée par les animaux.

La teneur en MAT des bols «pâturage » n'étant pas différente de celle des bols «auge » $\mathrm{du} \mathbf{1}^{\text {er }}$ cycle (tabl. 5), ni pour le mouton ni pour le poney, il semble qu'aucune des deux espèces n'ait effectué de tri lors du pâturage. En revanche, au $2^{\text {e }}$ cycle (tabl. 5), la teneur en MAT des bols "pâturage " est systématiquement plus élevée que celle des bols " auge". Les animaux au pâturage ont donc très certainement trié et ingéré une herbe plus riche en MAT que l'herbe fauchée et, cela, avec la même intensité pour les moutons et le poney.

La DIV et la digestibilité cellulase des bols «auge» sont plus élevées que la digestibilité mesurée in vivo;

- au $1^{\text {er }}$ cycle les valeurs DIV et digestibilité cellulase des "bols pâturage " (des moutons et du poney) sont voisines et de plus peu différentes de celles des " bols auge »;

- au $2^{c}$ cycle la digestibilité in vitro (DIV et cellulase) des bols de "pâturage " est significativement plus élevée que celle des bols « auge », la différence étant du même ordre de grandeur chez les moutons et le poney.

Les moutons et le poney auraient donc effectué le même tri au pâturage, ce qui confirme les observations relatives à la teneur en MAT.

Il est bien sûr impossible de généraliser ces résultats - qui vont d'ailleurs à l'encontre de l'aptitude, connue, du mouton à trier encore plus sélectivement que 


\section{TABleau 5}

Comparaison, pour les périodes fauche et pâturage correspondantes,

des teneurs en matières azotées totales (MAT) des bols et de l'herbe fauchée d'une part, de la digestibilité in vivo des bols d'autre part, suivant l'espèce animale et le numéro du cycle.

Comparison for indoor and grazing corresponding periods

between crude protein content of herbage and of extrusa on the one hand and on the other hand in vivo digestibility of cut forage

and in vitro digestibility of extrusa, according to animals and to cycle order.

\begin{tabular}{|c|c|c|c|c|c|c|}
\hline \multirow{2}{*}{$\begin{array}{l}\text { Animaux } \\
\text { Animals }\end{array}$} & \multirow{2}{*}{$\begin{array}{c}\text { Numéro } \\
\text { du cycle }\end{array}$} & \multirow{2}{*}{$\begin{array}{l}\text { Exploitation } \\
\text { de l'herbe } \\
\text { Herbage } \\
\text { exploitation }\end{array}$} & \multicolumn{2}{|c|}{$\begin{array}{l}\text { MAT (p. } 100 \mathrm{MS}) \\
C P(p .100 D M)\end{array}$} & \multicolumn{2}{|c|}{$\begin{array}{l}\text { Digestibilité MS (p. 100) } \\
\text { DM digestibility (p. 100) }\end{array}$} \\
\hline & & & $\begin{array}{c}\text { Herbe } \\
\text { fauchée } \\
\text { Cut herbage }\end{array}$ & $\begin{array}{c}\text { Bols } \\
\text { Extrusa }\end{array}$ & $\begin{array}{c}\text { In vivo } \\
\text { Herbe } \\
\text { fauchée } \\
\text { Cut herbage }\end{array}$ & $\begin{array}{c}\text { In vitro (T.T.) } \\
\text { Bol lyophilisé } \\
\text { Freeze dried } \\
\text { extrusa }\end{array}$ \\
\hline \multirow{4}{*}{$\begin{array}{l}\text { Mouton } \\
\text { Sheep }\end{array}$} & & $\begin{array}{l}\text { Auge } \\
\text { Indoor }\end{array}$ & 18,9 & 18,6 & 68,7 & 81,3 \\
\hline & $1 s t$ & $\begin{array}{l}\text { Pâturage } \\
\text { Grazing }\end{array}$ & - & 18,3 & - & 80,5 \\
\hline & $2^{\circ}$ & $\begin{array}{l}\text { Auge } \\
\text { Indoor }\end{array}$ & 13,0 & 13,0 & 58,5 & 70,0 \\
\hline & 2nd & $\begin{array}{l}\text { Pâturage } \\
\text { Grazing }\end{array}$ & - & 18,1 & - & 79,4 \\
\hline \multirow{4}{*}{$\begin{array}{l}\text { Poney } \\
\text { Poney }\end{array}$} & $1^{\text {er }}$ & $\begin{array}{l}\text { Auge } \\
\text { Indoor }\end{array}$ & 18,9 & 17,4 & 63,4 & 80,0 \\
\hline & $1 s t$ & $\begin{array}{l}\text { Pâturage } \\
\text { Grazing }\end{array}$ & - & 17,4 & - & 79,7 \\
\hline & \multirow{2}{*}{$\begin{array}{c}2^{e} \\
2 n d\end{array}$} & $\begin{array}{l}\text { Auge } \\
\text { Indoor }\end{array}$ & 13,0 & 13,3 & 53,6 & 66,1 \\
\hline & & $\begin{array}{l}\text { Pâturage } \\
\text { Grazing }\end{array}$ & - & 17,6 & - & 77,3 \\
\hline
\end{tabular}

le cheval, comme l'ont montré ARNold \& DUNZINSKI (1978) ou encore Loiseau, MARTIN-Rosset \& BECHET (1980) - compte tenu du nombre restreint de mesures et du fait que nous n'avions qu'un poney fistulé mis en parallèle avec deux types de chevaux différents, pouliches et trotteurs.

En définitive, dans nos essais, les deux espèces animales (mouton et cheva!) n'auraient pâturé sélectivement, et de la même façon, qu'au $2^{\circ}$ cycle et, cela, malgré une faible pression de pâturage (voisine de l'ad libitum) pour les deux cycles de végétation. 
- Comparaisons de la digestibilité de l'herbe pâturée estimée à partir soit des prélèvements œsophagiens soit des caractéristiques fécales :

La digestibilité réelle de l'herbe pâturée n'est pas connue. On ne peut donc juger la fiabilité et l'intérêt de différentes équations de prévision que sur leur précision (valeur de leur écart type réduit) et sur leur aptitude à conduire à des valeurs de dMO calculées les plus regroupées possibles (LANGLANDS, 1967).

Nous avons reporté dans le tableau 6 les valeurs de la digestibilité calculée à partir des 2 équations digestibilité in vitro et cellulase des bols lyophilisés et des 6 équations "caractéristiques fécales" permettant la meilleure précision (Syx $<2,4$ chez le mouton, $<2,9 \mathrm{chez}$ le cheval).

\section{TABLeau 6}

Valeurs estimées de la digestibilité de l'herbe pâturée calculées à partir soit de la technique des bols, soit des caractéristiques fécales.

Etimated value of grazed herbage digestibility calculated either from extrusa or from faecal characteristics.

\begin{tabular}{|c|c|c|c|c|c|}
\hline \multirow{3}{*}{$\begin{array}{c}\text { Espèce } \\
\text { animale } \\
\text { Animal } \\
\text { species }\end{array}$} & \multirow{3}{*}{$\begin{array}{c}\text { Numéro } \\
\text { du cycle } \\
\text { Cycle } \\
\text { order }\end{array}$} & \multirow{3}{*}{$\begin{array}{c}\text { Digestibilité } \\
\text { in vivo } \\
\text { de l'herbe fauchée } \\
\text { In vivo digestibility } \\
\text { of cut herbage }\end{array}$} & \multicolumn{3}{|c|}{$\begin{array}{l}\text { Disgestibilité calculée de l'herbe pâturée } \\
\text { Calculated digestibility of grazed herbage }\end{array}$} \\
\hline & & & \multicolumn{2}{|c|}{ Esophage - Oesophagus } & \multirow{2}{*}{$\begin{array}{l}\text { Fèces - Faeces } \\
\text { Valeurs extrêmes } \\
\text { Range of values }\end{array}$} \\
\hline & & & DIV & Cellulase & \\
\hline \multirow{2}{*}{$\begin{array}{l}\text { Mouton } \\
\text { Sheep }\end{array}$} & $\begin{array}{l}\text { Premier } \\
\text { First }\end{array}$ & 68,7 & $\begin{array}{l}\mathrm{L} 71,5 \\
\mathrm{E}-\end{array}$ & $\begin{array}{l}73,8 \\
-\end{array}$ & $\begin{array}{l}70,8-74,0 \\
n=6\end{array}$ \\
\hline & $\begin{array}{l}\text { Second } \\
\text { Second }\end{array}$ & 58,5 & $\begin{array}{l}\text { L } 70,8 \\
\text { E } 71,2\end{array}$ & $\begin{array}{l}71,1 \\
71,7\end{array}$ & $\begin{array}{l}68,7-73,6 \\
\mathrm{n}=6\end{array}$ \\
\hline \multirow{2}{*}{$\begin{array}{l}\text { Poney } \\
\text { Pony }\end{array}$} & $\begin{array}{l}\text { Premier } \\
\text { First }\end{array}$ & 63,4 & $\begin{array}{l}\text { L } 52,1 \\
\text { E } 75,0\end{array}$ & $\begin{array}{l}69,1 \\
58,8\end{array}$ & $\begin{array}{l}55,1-73,2 \\
\mathrm{n}=6\end{array}$ \\
\hline & $\begin{array}{l}\text { Second } \\
\text { Second }\end{array}$ & 53,6 & $\begin{array}{l}\text { L } 57,9 \\
\text { E } 71,6\end{array}$ & $\begin{array}{l}64,4 \\
58,7\end{array}$ & $\begin{array}{l}55,0-64,0 \\
\mathrm{n}=6\end{array}$ \\
\hline
\end{tabular}

Les valeurs de la digestibilité, calculées à partir des prélèvements œsophagiens et des caractéristiques fécales, étant comparables chez le mouton, l'avantage revient à ces dernières qui conduisent à une erreur de prévision plus faible ( 2 à 2,5 points de digestibilité) que les prélèvements œsophagiens (3,3 à 4,1 points de digestibilité). Chez le cheval, ces valeurs sont en revanche très dispersées pour chacune des deux techniques et il est difficile de porter un jugement sur leur intérêt relatif d'autant plus 
que le nombre de mesures effectuées au pâturage a été limité. L'avantage reviendrait aux caractéristiques fécales pour leur degré de précision plus élevé. Il conviendrait toutefois, à l'avenir, de préciser celles de ces caractéristiques conduisant à la dispersion des valeurs de digestibilité calculées la moins grande.

\section{Conclusion}

Malgré les nombreux travaux publiés depuis trente ans sur la prévision de la digestibilité de l'herbe pâturée et les quantités qui en sont ingérées, beaucoup de problèmes méthodologiques restent encore à éclaircir (LANGLANDS, 1975 ; LE DU \& Penning, 1982). Les résultats de nos essais où nous avons utilisé 3 espèces animales (mouton, cheval, bovins) et les prélèvements œsophagiens et les caractéristiques fécales, permettent d'avancer les conclusions suivantes :

\section{A. Fistule de l'oesophage}

Les sources d'erreurs sont nombreuses (LANGLANDS, 1975) et les plus importantes proviennent :

- du mode de séchage des bols entiers, car il ne semble pas nécessaire de séparer la salive. Le séchage par lyophilisation ne permet pas forcément une meilleure homogénéité des résultats que celui à l'étuve qui peut également être retenu dans certains cas ;

- de la dispersion irrégulière des valeurs DIV ou digestibilité cellulase des bols par rapport à celles de l'échantillon de l'herbe offerte à l'auge correspondant.

Il convient donc, à notre avis, d'être extrêmement prudent dans l'exploitation des résultats de digestibilité in vitro ou cellulase. Selon nos résultats il est impossible d'utiliser directement ces derniers comme valeur réelle de la digestibilité de l'herbe pâturée. Nous devons donc utiliser une équation de régression intermédiaire qui est cependant entachée d'une erreur résiduelle élevée (Syx ; 3,31 et 5,14) pour la DIV (bol lyophilisé et étuvé respectivement) et 3,66 et 4,66 (bol séché à l'étuve) pour la digestibilité cellulase, respectivement chez le mouton et le cheval. Mais cela nécessite de conduire simultanément un essai à l'auge comme dans le cas des techniques "fèces". Cette conclusion, pessimiste, supprimerait tout l'intérêt de la technique de la fistule de l'œsophage, même pour déterminer la composition botanique de l'herbe pâturée qui peut être facilement obtenue à partir de l'analyse des cuticules et épidermes contenus dans les fèces.

\section{B. Caractéristiques fécales}

En utilisant des caractéristiques pariétales ou physiques en plus de l'azote fécal (classique) ou des matières fécales solubles, il est possible d'établir des équations de régression utilisables pour l'ensemble des cycles de repousse. La précision de ces équations est bonne puisqu'elle est comprise entre 2 et 3 points de digestibilité 
pour le cheval et le mouton. Toutefois elles ne conduisent à des valeurs de digestibilité calculées homogènes que dans le cas du mouton.

\title{
C. Valeur relative des deux types d'équations
}

Sur la base du classement relatif et de la précision de la prévision des digestibilités: calculées, l'avantage va, chez le mouton, aux caractéristiques fécales (tabl. 4). Chez le cheval et les bovins il aurait été intéressant de réaliser des essais plus nombreux au pâturage et de fistuler les trotteurs (mesures à l'auge) et des pouliches (mesures au pâturage). Enfin, il aurait été préférable de mesurer la DIV des bols cheval avec du jus de caecum au lieu de jus de rumen. On sait en effet (Koller et al., 1978) que l'activité cellulolytique des micro-organismes du rumen est plus élevée que celle du caecum, ce qui a vraisemblablement introduit une source d'erreur dans nos mesures sur les bols de poney.

Quoiqu'il en soit, les 2 groupes d'équations bols et fèces retenus chez le mouton permettent de classer les digestibilités suivant leur valeur relative et d'apprécier l'intensité du tri au pâturage par rapport à l'affouragement en vert. Elles permettent aussi de mettre en évidence, même au pâturage, la capacité digestive plus élevée chez le ruminant que chez le cheval.

Ces essais ont été réalisés sur une prairie naturelle plurispécifique à forte production et de bonne qualité. Il conviendrait de vérifier ces résultats dans le cas de parcours extensifs plus pauvres et moins productifs.

Reçu en juin 1985.

Accepté en décembre 1985.

\section{Remerciements}

Nous tenons à remercier J. Lefaivre qui a réalisé les interventions chirurgicales nécessaires à la pose des canules de l'œsophage sur moutons, vaches et surtout poney.

Nous remercions également Jocelyne Aufrere pour les mesures de digestion à la cellulase des bols de l'œsophage.

\author{
Summary \\ Methodological aspects of predicting herbage digestibility \\ in grazing sheep, cattle and horses using oesophageal fistula \\ or faecal index techniques
}

Some methodological aspects of herbage digestibility estimation were analysed in grazing sheep, cattle and horses using either oesophageal or faecal characteristics.

A preliminary trial (Chenost, Martin-Rosset, 1985) was made to compare the digestive capacity of sheep, cattle and horses fed green herbage. Three trials with various forages 
(table 1) were made in 1980, 81 and 82 . Characteristics of these forages and animals used (6 sheep, 5 horses, 6 dairy cows and 6 young bulls) are reported in table 1 .

Parallel to digestibility and feed intake measurements during indoor trials, a grazing trial was carried out in 1981: two 2-year old mares and 6 castrated sheep grazed simultaneously the 1 st and 2 nd cycle regrowths of a natural pasture. The grazing pressure was the same for both cycles. It was not possible to include cattle in the mixed sheep and horse grazing trial. In both indoor and grazing trials, one fistulated poney and 2 fistulated sheep were added to the other animals; 2 fistulated 2-year old heifers were added to the cattle group during the indoor trial only.

During each indoor digestibility trial, oesophageal extrusa were obtained by three 30-minute collections (on Mondays, Wednesdays and Fridays) during the two main meals from the previously fasted fistulated animals. In grazing trials extrusa were collected from fasted animals grazing the pasture three days a week for $30 \mathrm{~min}$ in the morning.

Various methods for a) collecting and b) processing extrusa were tested :

- collection with or without obturating the oesophagus ;

- squeezing or not extrusa in a muslin for eliminating saliva;

- oven-drying of extrusa at $80{ }^{\circ} \mathrm{C}$ for $48 \mathrm{~h}$ or freeze-drying.

Faeces were collected every day during the indoor digestibility trial and three times a week (Mondays, Wednesdays, Fridays) during grazing trials. Sheep faeces were collected using bags and harnenesses, mare faeces were grab sampled.

The following measurements were carried out:

- extrusa : dry matter (DM), ash, crude protein contents (CP), in vitro digestibility (IVD, Tilley-Terry) and cellulase digestibility (cellulase).

- faecal samples : a) chemical assays involving DM, crude protein (FCP) and water soluble contents (FWSM) for non cell-wall constituents; crude fibre content (FCF), NDF and ADF Van Soest, for cell-wall components; b) physical measurements involving specific gravity (SG), JARRige, 1965 and particle size (PS), E. GrENET, 1966 on ground faeces.

As regards extrusa collection, results showed that oesophagus obturation was not required. DM recovered through fistulae (in p. 100 of DM intake) was not increased. Even when oesophagus was not obturated the recovery rate was lower than that generally observed, probably because animals were not yet accustomed to manipulations. Later on, we recovered $300-500 \mathrm{~g}$ extrusa within 20 minutes. The recovery rate was higher in horses than in sheep (table 2). Extrusa DM was higher in horses than in ruminants.

Saliva removal did not improve the repeatability of in vitro digestibility (IVD) and cellulase results. Adding the removed saliva to the initial proportion for IVD and cellulase measurements did not improve IVD (table 2). The drying technique did not affect either ash or CP content of extrusa.

IVD or cellulase results of oven-dried extrusa were lower than those obtained after freeze-drying (fig. 1 and 2). In both cases the variability was large as well as the difference between oven and freeze drying for both IVD and cellulase. IVD of extrusa was higher than cellulase digestibility of extrusa. These extrusa digestibilities were both higher than a) the IVD and cellulase digestibility and b) the in vivo digestibility of the cut herbage (fig. 2). On the basis of these data it is therefore not possible to directly derive any in vivo digestibility figure for grazed herbage. Accordingly, it is necessary to use an intermediate regression equation for estimating the in vivo digestibility of grazed herbage (table 3).

Correlations between in vivo digestibility of green herbage and different faecal characteristics are reported in table 4. Faecal characteristics are considered either as one variable or as a couple of two variables. Standard errors Syx were promising as they ranged between 2 and 3 points of digestibility. These correlations were calculated for all the indoor data without taking into account the plant species or regrowth cycle order. Table 4 gives the range of the regression equations with a decreasing accuracy.

The degree of plant selection was appreciated in sheep and horses (table 5) by comparing extrusa composition (CP and IVD) in both grazing trials. Sheep and horses did not select during the first cycle, while they selected during the second one, in spite of a similar grazing pressure during both cycles. 
On the basis of results reported in table 6 we analysed data obtained in deriving estimates of grazed herbage digestibility from the two categories of equations (fistulation and faecal techniques). As compared to fistulation results, those obtained with faecal techniques were more homogeneous and prediction was more precise.

\section{Références bibliographiques}

Arnold G.W., Dudzinski M.L., 1978. Ethology of free ranging domestic animals. Elsevier Scientific Publishing Company, Amsterdam, p. 97.

Aufrere J., 1982. Etude de la prévision de la digestibilité des fourrages par une méthode enzymatique. Ann. Zootech., 31, 111-130.

Chenost M., 1985. Estimation de la digestibilité de l'herbe ingérée au pâturage à partir de l'azote fécal et de quelques autres paramètres fécaux. Ann. Zootech., 34, 205-228.

Chenost M., Martin-Rosset W., 1985. Comparaison entre espèces (mouton, cheval, bovin) de la digestibilité et des quantités ingérées de fourrages verts. Ann. Zootech., 34, 291-312.

Grenet E., 1966. Les particules végétales des fèces de moutons. Ann. Zootech., 16, 303-312.

JARRIGE R., 1965. The composition of sheep faeces and its relation to forage digestibility. Proc. 9th Intern. Grasst. Congr., 389, 809-814.

Koller B.L., Hintz H.F., Robertson J.B., van Soest P.J., 1978. Comparative cell wall and dry matter digestion in the caecum of the poney and the rumen of the cow using. in vitro and nylon bag techniques. J. Anim. Sci., 47, 209-215.

LANGLANDS J.P., 1967. Studies on the nutritive value of the diet selected by grazing sheep. III. A comparison of oesophageal fistula and faecal index techniques for the indirect estimation of the digestibility. Anim. Prod., 9, 325-331.

LANGLANDS J.P., 1975. Techniques for estimating nutrient intake and its utilization by the grazing ruminants. In : McDonald I.W. et Warner A.C.I. (Eds). Digestion and metabolism in the ruminant, pp. 320-332.

LE Du Y.L.P., Penning P.G., 1982. Animal based techniques for estimating herbage intake. In : Leave: J.D. (Ed.). Herbage intake handbook. The British Grassland Soc. (Publ.), $37-75$.

Loiseau P., Martin-Rosset W., Bechet G., 1980. Effect of domestic herbivores and botanical composition of hill pastures. European Grassland Federation, Zagreb Meeting, Session VII, 79-86.

MEIJS J.A.C., 1981. In : Herbage intake by grazing dairy cows. Institute for Livestock Feeding and Nutrition Research «Hoorn », Lelystad, Wageningen, 28-31.

Streeter C.L., 1969. A review of techniques used to estimate the in vivo digestibility of grazed forage. J. Anim. Sci., 28, 119-128.

Tilley J.A.M., Terry R.A., 1963. A two stage technique for in vitro digestion of forage crops. J. Br. Grassl. Soc., 18, 101-111.

Tissier M., Bechet G., Molenat G., 1975. Appareils de collecte totale des fèces pour agneaux en allaitement ou à l'engrais et pour brebis. Ann. Zootech., 24, 595-602.

VAN SOEST P.J., 1967. Development of a comprehensive system of feed analyses and its applications to forages. J. Anim. Sci., 28, 119-128. 